\title{
Neue Webinar-Reihe „Go for IT“: Handwerkszeug, das es sich zu lernen lohnt
}

"Go for IT"

\section{die neue Webinar-Reihe der Akademie Online}

Big Data, Radiomics, Künstliche Intelligenz - diese IT-Themen sind in der radiologischen Community derzeit heiß diskutiert und stehen bei einer Vielzahl aktueller Fortbildungsveranstaltungen und Kongressen auf dem Programm. Zu Recht, denn sie gehen uns alle an - schließlich dürfte von ihnen abhängen, wie unsere Radiologie der Zukunft aussehen wird.

Doch was steht eigentlich hinter solchen Begriffen wie „Big Data“ oder „Radiomics“? Wissen wir, wovon wir sprechen, wenn wir diese Begriffe benutzen, und sprechen wir eigentlich alle über dasselbe? Wie muss die Datenbasis von KI-Systemen aussehen, um aussagefähige Ergebnisse für den Patientennutzen zu bringen? Mit welchen Datensätzen und Algorithmen werden Radiologien künftig zu tun haben?

Es ist Zeit, in diesem Bereich „eine gemeinsame Sprache zu schaffen“, finden PD Dr. Bettina Baeßler von der Uniklinik Mannheim und Dr. Daniel Pinto dos Santos von der Uniklinik Köln. Sie sind Initiatoren eines neuen Webinar-Angebots, das unter dem Titel „Go for IT“ allen Interessierten die entsprechenden IT-Backgrounds vermitteln will, und zwar Hands On! In den Webinaren soll es um die Statistik und die Programmierung gehen, ohne die „Big Data“ und „Radiomics“ nur leere Begriffe wären, und das im Wechsel von Theorie und Praxis: In jedem zweiten Webinar steigen die Teilneh- mer direkt in die Programmierung der Statistik-Software "R“ ein; diese ist frei verfügbar (s. u.). „Das ist Handwerkszeug, das es sich zu lernen lohnt!“, erklärt Dr. Pinto dos Santos zur Vorankündigung der Reihe, die sich an alle (angehenden) Radiologinnen und Radiologen richtet. Jeder, der mehr über das Thema wissen und praktische Erfahrungen sammeln möchte, kann an den Online-Kursen teilnehmen. Die Teilnahme ist kostenfrei, Vorkenntnisse im Bereich der Programmierung sind nicht erforderlich.

Mehr Informationen: www.drgakademie.de $>$ Akademie Online > Go for IT

\section{Das Programm 2019 im Überblick:}

17.06.2019 Go for IT 3: Grundzüge in Statistik: sicher signifikant

08.07.2019 Go for IT 4: Nächste Schritte in R: bunte Bilder und mehr

23.09.2019 Go for IT 5: Wer Test sagt kann auch p sagen? Statistik überall

14.10.2019 Go for IT 6: Theoretisch ja, praktisch auch! Tests in $\mathrm{R}$

11.11.2019 Go for IT 7: Genug gelernt, jetzt sind die Maschinen dran! Grundzüge Machine Learning

09.12.2019 Go for IT 8: See one, do one, teach one! Machine Learning in $\mathrm{R}$

Eine Fortführung der Reihe mit weiteren Terminen in 2020 ist in Planung.

\section{„GO FOR IT“ BEIM RÖKO IN LEIPZIG}

Bei diesem Live-Termin beim RöKo haben Sie die Gelegenheit, mit den Referenten und anderen Teilnehmern in einen interaktiven Austausch zu treten und Ihre Fragen zum Thema persönlich zu diskutieren! Vorab bietet der Kurs all denjenigen Interessierten einen leichten Einstieg, die die bisherigen Webinare verpasst haben oder einfach gerne nochmal wiederholen möchten. Teilnehmer sollten einen eigenen Laptop mitbringen und idealerweise bereits vor dem Kurs die Software R (https://cran.rstudio.com) und die graphische Oberfläche RStudio (https:// www.rstudio.com) installieren.

FR, 31.05.2019, 9:45-10:45 Uhr, Raum Levy Dorn

9:15 - 10:15: Einführung in aktuelle IT-Themen, Statistik und Programmierung mit R (Zusammenfassung/Wiederholung der ersten beiden WebinarTermine)

10:15 - 10:45: Diskussion, Fragen und Wünsche 